\title{
Leptin Contributes to Slower Weight Gain in Juvenile Rodents on a Ketogenic Diet
}

\author{
LIU LIN THIO, EBRU ERBAYAT-ALTAY, NICHOLAS RENSING, AND KELVIN A. YAMADA
}

\begin{abstract}
Departments of Neurology [L.L.T., E.E.-A., N.R., K.A.Y.] and Pediatrics [L.L.T., K.A.Y.] and the Hope Center for Neurological Disorders [L.L.T., E.E.-A., N.R., K.A.Y.], Washington University School of Medicine, St. Louis, Missouri 63110; Department of Pediatric and Developmental Neurology [L.L.T., K.A.Y.] and the Pediatric Epilepsy Center [L.L.T., K.A.Y.], St. Louis Children's Hospital, St. Louis, Missouri 63110
\end{abstract}

\begin{abstract}
The ketogenic diet (KD) is an efficacious therapy for medically refractory childhood epilepsy that also slows weight gain. We tested the hypothesis that the KD slows weight gain via neurohormones involved in energy homeostasis. We found that juvenile rodents fed a KD had slower weight gain than those fed a standard diet (SD). Rats fed a KD had higher serum leptin levels and lower insulin levels compared with those fed an SD. We investigated the increase in leptin further because this change was the only one consistent with slower weight gain. Although rats fed the SD experienced slower weight gain when calorie restricted, they had serum leptin levels similar to those fed the SD ad libitum. Furthermore, leptin deficient $(o b / o b)$ and leptin receptor deficient $(d b / d b)$ mice did not show slower weight gain on the KD. All animals on the KD had elevated serum $\beta$-hydroxybutyrate $(\beta \mathrm{HB})$ levels. Thus, ketosis is insufficient and a functioning leptin signaling system appears necessary for the KD to slow weight gain. The increase in leptin may contribute to the anticonvulsant effects of the KD. (Pediatr Res 60: 413-417, 2006)
\end{abstract}

$\mathrm{F}^{-1}$ ew treatment options exist for the 20-30\% of epileptic children whose seizures are refractory to medication. One option is the $\mathrm{KD}$, a high-fat, low-carbohydrate, and adequate protein diet. The KD is remarkably effective in children with medically refractory epilepsy as $5-10 \%$ become seizure free and $30 \%$ have a $>90 \%$ reduction in seizure frequency (1). Yet, its adverse effects and the parental effort involved in implementing and maintaining the diet limit its use. Thus, all epileptic children would benefit from a simpler method of attaining the diet's anticonvulsant effects, which requires elucidating its mechanism of action.

Here we focus on what the impaired weight gain associated with the KD might reveal about the diet's anticonvulsant mechanism. Several studies report that children on the KD drop about 10 percentiles in weight, although appropriate

Received January 12, 2006; accepted May 15, 2006.

Correspondence: Liu Lin Thio, M.D., Ph.D., Department of Neurology, Washington University School of Medicine, 660 South Euclid Avenue, Box 8111, St. Louis, MO 63110; e-mail: thiol@neuro.wustl.edu

Supported by grants from the National Institutes of Health (NIH) (DK 20579, NS 42774), the Juvenile Diabetes Research Foundation (1-2004-594), and the Washington University McDonnell Center for Cellular and Molecular Neurobiology. The Washington University Diabetes Research and Training Center (NIH DK 20579) and the Washington University Clinical Nutrition Research Center (NIH DK 56341) also provided assistance. This work was performed in a facility supported by the NIH (NCRR C06 RR015502).

DOI: 10.1203/01.pdr.0000238244.54610.27 weight gain is the goal $(2,3)$. Recognizing that lowcarbohydrate diets, such as the Atkins diet, are ketogenic makes this impaired weight gain less surprising. Indeed, the Atkins diet and other low-carbohydrate KDs result in a $5 \%$ to $10 \%$ weight loss over 6 mo $(4,5)$. Furthermore, rodents placed on a KD show slower weight gain $(6,7)$.

We hypothesized that the anticonvulsant and weight effects of the KD share a common mechanism involving a change in the serum levels of leptin, insulin, ghrelin, or cortisol. These peripherally released hormones help determine body weight because they regulate energy homeostasis (8). Importantly, leptin and insulin also modulate neuronal excitability $(9,10)$.

\section{METHODS}

Dietary protocols. Experimental protocols were approved by the Washington University Animal Studies Committee. Sprague-Dawley rats (Charles River Laboratories, Wilmington, MA), male ob/ob mice (Stock 000632, The Jackson Laboratory, Bar Harbor, ME), male C57BL/6J mice (Stock 000664, The Jackson Laboratory), male $d b / d b$ mice (Stock 000642, The Jackson Laboratory), and male C57BLKS/J mice (Stock 000662, The Jackson Laboratory) were housed under a 12-h light/dark cycle. Experiments with rats began on postnatal day (PD) 21 , the day of weaning, and those with mice began when $o b / o b$ and $d b / d b$ mice are phenotypically identifiable. Cages held four to six rats or two to three mice. All animals had ad libitum access to water. They were fed a SD (Rodent Diet 20, PicoLab, Richmond, IN), a KD (F3666; Bio-Serv, Frenchtown, NJ), or a calorie-restricted diet (CD). Animals on the SD received $12 \%$ of their calories from fat, $65 \%$ from carbohydrate, and $24 \%$ from protein. By weight, the SD was $20 \%$ protein, $5 \%$ fat, $5 \%$ fiber, $55 \%$ carbohydrate, and $6 \%$ ash. Animals on the KD received $92 \%$ of their calories from fat, $3 \%$ from carbohydrate, and $5 \%$ from protein. By weight, the KD was $8 \%$ to $9 \%$ protein, $75 \%$ fat (45\% lard, $19 \%$ butter, and $10 \%$ corn oil), $4 \%$ fiber, $3 \%$ ash, $4 \%$ to $6 \%$ carbohydrate, $<10 \%$ moisture, and $2 \%$ vitamin. The KD had a 6:1 ratio of fat to carbohydrate + protein by weight. Animals on the SD and the KD had ad libitum access to chow. Animals on the $\mathrm{CD}$ received about $40 \%$ of the calories that those on the SD received. Animals on the $\mathrm{CD}$ were fed at 11:00 a.m., and they consumed all allotted chow in two hours. Caloric intake was calculated by weighing the chow consumed by one cage daily and assuming all animals had the same caloric intake when normalized to body weight. Animals were killed on PD 26 to 50 between 1000 and $1400 \mathrm{~h}$. Although leptin and insulin are at their nadir at this time, their diurnal variation is markedly diminished with fasting and that of leptin is markedly diminished with high-fat diets (11-13). Blood was collected by a right ventricular cardiac tap.

Dual energy $x$-ray absorptiometry (DEXA) scans. The percentage body fat was obtained by scanning the torso and limbs of rats using the PIXImus

Abbreviations: $\boldsymbol{\beta} \mathrm{HB}, \boldsymbol{\beta}$-hydroxybutyrate; $\mathrm{CD}$, calorie restricted diet; $\boldsymbol{d} \boldsymbol{b} / \boldsymbol{d} \boldsymbol{b}$, homozygous leptin receptor deficient mice; DEXA, dual energy x-ray absorptiometry; KD, ketogenic diet; $\boldsymbol{o b} / \boldsymbol{o b}$, homozygous leptin deficient mice; PD, postnatal day; SD, standard diet 
densitometer and software (GE Lunar, Madison, WI) under ketamine (40 $\mathrm{mg} / \mathrm{kg}$ ) and xylazine $(10 \mathrm{mg} / \mathrm{kg})$ anesthesia.

Assays. Serum glucose concentrations were measured using a glucometer. Serum $\beta \mathrm{HB}$ concentrations were determined enzymatically except as shown in Figure 3 in which the KetoSite Test Kit (Stanbio Laboratory, Boerne, TX) and STAT-Site analyzer (GDS Diagnostics, Elkhart, IN) were used. Enzymatically determined values typically were higher. Serum cortisol, ghrelin, insulin, and leptin levels were determined by radioimmunoassay (DiaSorin S.p.A., Stillwater, MN or Linco Research, Inc., St. Charles, MO).

Data analysis. Plots of body weight or normalized caloric intake versus PD were compared using a repeated-measures analysis of variance (ANOVA) (SPSS, Chicago, IL). Means were compared by a $t$ test or ANOVA with Tukey's post hoc comparison of means (OriginLab, Northampton, MA). Linear fits were obtained by linear regression. Data are presented as mean \pm standard error of the mean (SEM). Statistical significance was set at $p<0.05$.

\section{RESULTS}

Juvenile Sprague-Dawley rats gained weight more slowly on $\boldsymbol{a} \mathbf{K D}$. We fed juvenile Sprague-Dawley rat littermates a $\mathrm{KD}$ or SD for $2 \mathrm{wk}$. We chose juvenile rats because younger animals are better adapted for ketosis (14). The KD chow was the same chow used in several other rodent studies $(6,7,15)$. Rats tolerated the KD without difficulty. They appeared as healthy and active as their littermates fed the SD.

The littermates on the KD gained weight significantly more slowly than their siblings fed the SD as noted previously $(6,7)$ (Fig. 1A). Heart, liver, and kidney wet weights were $33 \%$ to $48 \%$ lower while brain mass was preserved after 2 wk on the $\mathrm{KD}$ (Fig. $1, B-E$ ). Aside from differences in mass, organs from
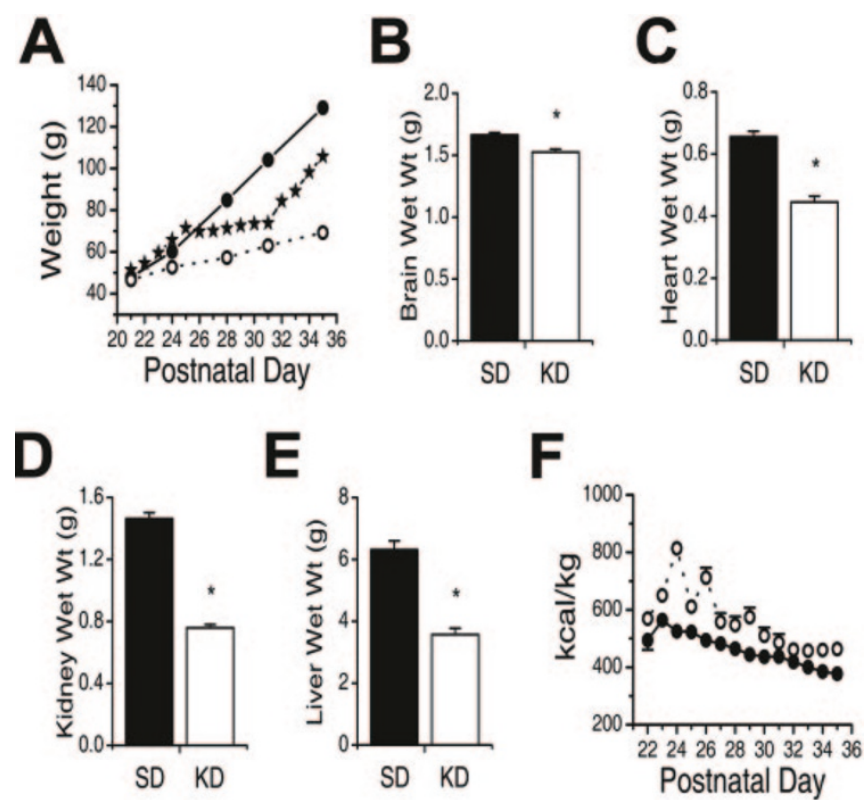

Figure 1. Rats fed a KD show slower weight gain. (A) Weight curves for rats on the SD (filled circles, $n=25$ ) from PD 21 to PD 35, the KD (open circles, $n=26$ ) from PD 21 to PD 35, or the KD from PD 26 to PD 30 (stars, $n=$ 12). Symbols show mean body weight. Weight curves for rats on the SD and KD from PD 21 to PD 35 differ $(p<0.05)$. Normally growing rats on the SD show slower weight gain when placed on the KD and resume normal weight gain upon return to the $\mathrm{SD}$ (stars). $(B-E)$ Mean organ wet weights at PD 35 from rats fed the $\mathrm{SD}$ (filled columns, $n=25$ ) or the $\mathrm{KD}$ (open columns, $n=$ 26) since PD 21. $* p<0.05 v s \mathrm{SD}$. (F) Curves for caloric intake on the SD $(n$ $=7)$ and $\mathrm{KD}(n=11)$ differ $(p<0.05)$. Symbols show mean caloric intake normalized to body weight and correspond to diets in $A$. Data in $A-E$ are from a different experiment than the data in $F$. Error bars show SEM and are shown in $A$ and $F$ if larger than the symbol. rats fed the KD were not grossly different from those fed the SD except for the liver being fatty. To determine whether the effect of the KD on body weight was reversible, we placed rats on the SD from PD 21 to PD 25, the KD diet from PD 26 to PD 30, and the SD from PD 31 to PD 35. These animals had a slower growth rate while on the KD that reversed on return to the SD (Fig. 1A). Interestingly, rats on the KD had higher normalized caloric intakes than those on the SD (Fig. 1F). Thus, the KD reversibly slowed weight gain including that of lean body mass in juvenile rats while maintaining brain growth.

Increased serum leptin in juvenile Sprague-Dawley rats fed $\boldsymbol{a} K \boldsymbol{K D}$. One mechanism by which the KD could slow weight gain is by altering serum leptin, insulin, ghrelin, and cortisol levels, which are hormones involved in regulating energy homeostasis (8). We hypothesized that the KD slows weight gain by increasing leptin, increasing insulin, decreasing ghrelin, or decreasing cortisol levels.

Rats on the KD for 2 wk had 10-fold higher midday serum levels of $\beta \mathrm{HB}$ and $30 \%$ lower glucose levels than rats on the SD (Fig. $2 A$ and $B$ ). The elevated $\beta$ HB levels indicated that the KD produced ketosis. The lower glucose was expected since low carbohydrate diets can lower blood glucose in humans $(5,16)$. Rats on the KD also had $140 \%$ higher leptin levels, $72 \%$ lower insulin levels, and slightly increased cortisol levels but similar ghrelin levels at midday compared with rats on the SD (Fig. $2 C-F$ ). Five days on the KD produced smaller increases in serum $\beta \mathrm{HB}$ and leptin than $2 \mathrm{wk}$ on the $\mathrm{KD}$, with the changes being significant and reversing upon return to the SD (Fig. $2 A$ and $F$ ). Although low-protein diets also slow weight gain, decrease insulin levels, and can increase leptin levels $(17,18)$, they increase ghrelin levels $(19)$, suggesting that our results do not arise solely from the lowprotein content of the KD. Of the hormonal changes observed, only the increase in leptin could slow weight gain.

We examined how serum $\beta \mathrm{HB}$ and leptin varied with weight because of the known relationship between these parameters. Obese humans fed a low-carbohydrate diet experience greater weight loss with higher $\beta$ HB levels (4). For both the $\mathrm{SD}$ and the $\mathrm{KD}$, the rats with the highest $\beta \mathrm{HB}$ levels on the day of sacrifice weighed the least (Fig. $2 G$ ). In rodents, serum leptin levels correlate with body mass index (20). Although weight and leptin levels did not correlate in rats on the SD, rats having higher leptin levels on the KD weighed more (Fig. $2 H)$. For both the SD and the KD, neither insulin nor the leptin:insulin ratio correlated with weight.

Serum leptin does not increase in juvenile SpragueDawley rats fed a CD. In another experiment, we compared the weight growth curves and serum leptin levels in rats fed a $\mathrm{KD}, \mathrm{CD}$, or $\mathrm{SD}$. We fed juvenile rat littermates a $\mathrm{KD}, \mathrm{CD}$, or $\mathrm{SD}$ for 2 wk. Rats on the KD and $\mathrm{CD}$ gained weight more slowly than their siblings fed the SD (Fig. 3A). The fat mass of rats on the $\mathrm{KD}$ as a percentage of total mass was two to three times that of the rats on the SD or CD (Fig. 3B). Rats on the KD had sixfold higher $\beta \mathrm{HB}$ than rats fed either the SD or $\mathrm{CD}$ (Fig. 3C). Relative to the SD, the changes induced by the $\mathrm{KD}$ and $\mathrm{CD}$ in leptin levels correlated with the changes in fat mass (Fig. 3D). Rats on the $\mathrm{CD}$ had access to chow just before 

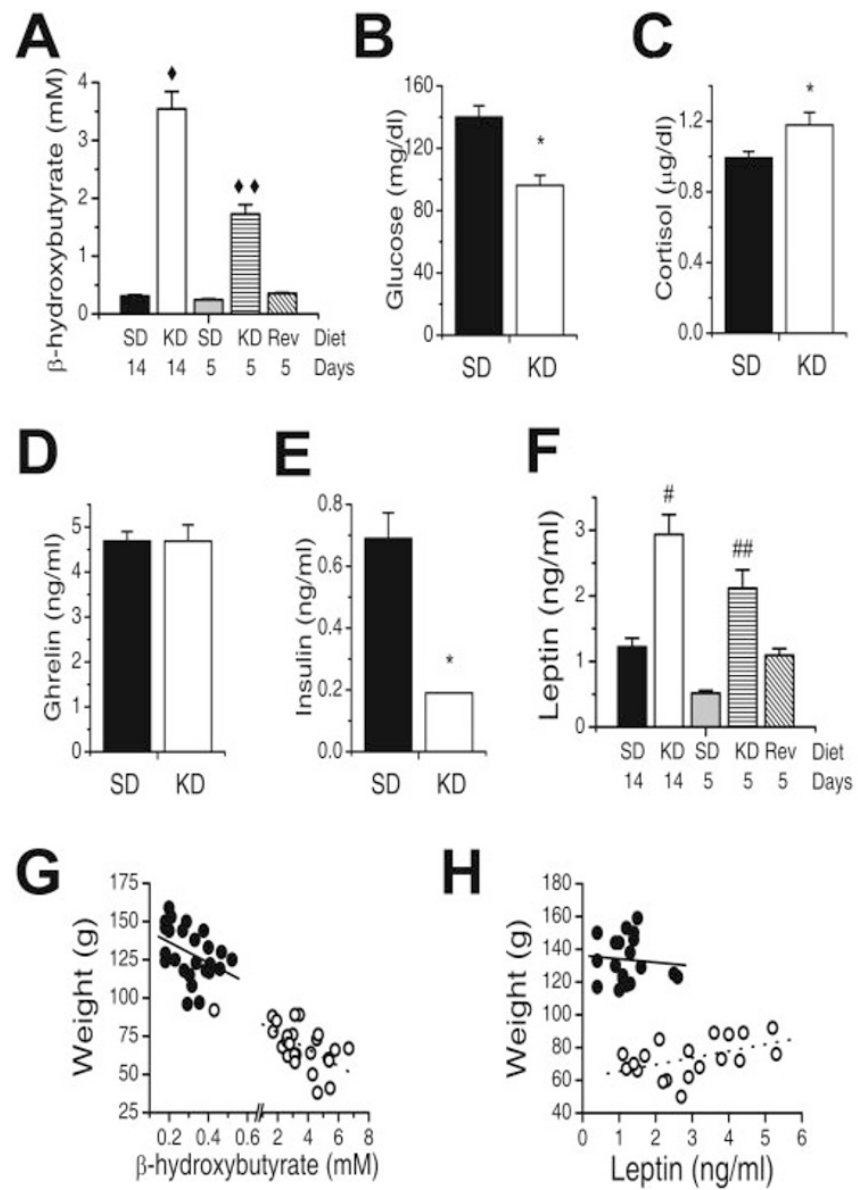

Figure 2. KD induced metabolic and hormonal changes. Mean serum $\beta \mathrm{HB}$ $(A)$, glucose $(B)$, cortisol $(C)$, ghrelin $(D)$, insulin $(E)$, and leptin $(F)$ levels in PD 35 rats fed the SD for $14 \mathrm{~d}$ (filled columns, $n=11-25$ ), PD 35 rats fed the KD for $14 \mathrm{~d}$ (open columns, $n=14-25$ ), PD 26 rats fed the SD for $5 \mathrm{~d}$ (shaded columns, $n=6-12$ ), PD 26 rats fed the KD for $5 \mathrm{~d}$ (horizontally striped columns, $n=6-14)$, or PD 36 rats fed the KD from PD 26-30 (labeled Rev, diagonally striped columns, $n=10-23$ ). $\bullet \bullet p<0.05 v s$ all other columns. ${ }^{*} p<0.05$ vs SD. \#p $<0.05$ vs left, middle, and right columns. \#\#p $<0.05$ vs middle column. Error bars show SEM. PD 35 weight $v s$ PD 35 serum $\beta \mathrm{HB}(G)$ and leptin $(H)$ in rats fed the SD (filled circles) or the KD (open circles) for $14 \mathrm{~d}$. Each point represents one rat. The lines in $G$ show a linear fit for the SD (solid line, $R=-0.40, p=0.045$ ) and the KD (dotted line, $R=-0.58, p=0.002$ ). The lines in $H$ show a linear fit for the $\mathrm{SD}$ (solid line, $R=-0.09, p=0.72$ ) and the $\mathrm{KD}$ (dotted line, $R=0.47, p$ $=0.04)$.

sacrifice, as did the rats on the SD and KD. For the rats on the $\mathrm{CD}$, access to chow just before sacrifice should produce maximal leptin levels (11) and may decrease $\beta \mathrm{HB}$ levels. These results demonstrate that the increase in serum $\beta \mathrm{HB}$ and leptin induced by the KD does not result from slower growth.

Juvenile ob/ob and db/db mice did not gain weight more slowly on a KD. We further examined the importance of leptin and $\beta \mathrm{HB}$ to the slower weight gain induced by the KD by using leptin deficient $o b / o b$ and leptin receptor deficient $d b / d b$ mice, which are both obese. If the KD requires leptin to slow weight gain, then it should not slow weight gain in $o b / o b$ and $d b / d b$ mice. Juvenile $o b / o b$ and $d b / d b$ mice fed the KD for 12-14 d gain weight at the same rate as $o b / o b$ and $d b / d b$ mice fed the SD (Figs. $4 A$ and 5A). These results suggest that the lower protein content and possible lower palatability of the
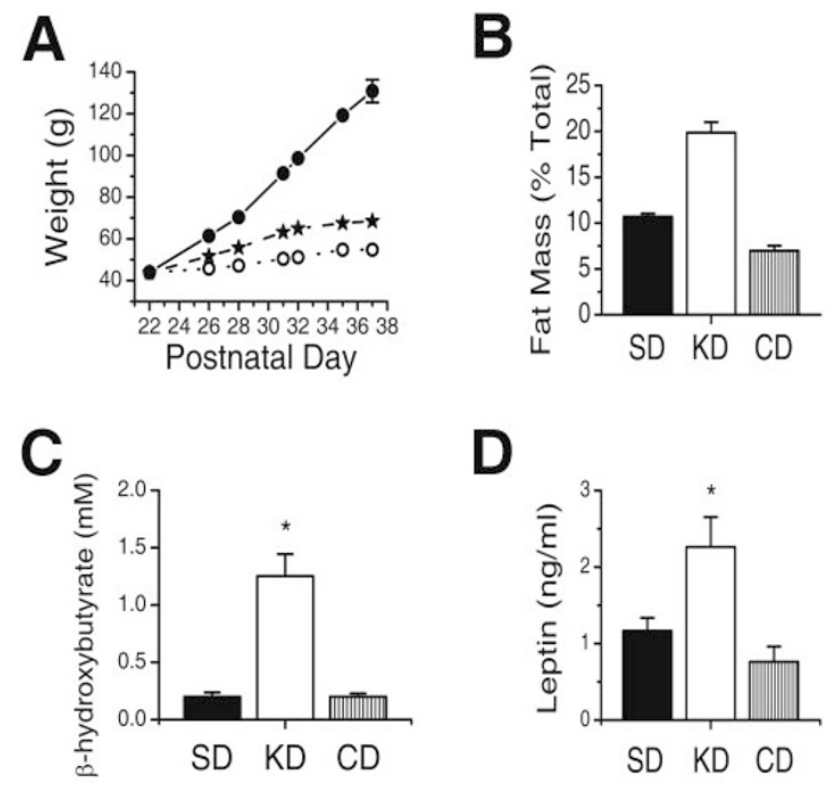

Figure 3. $\mathrm{CD}$ does not increase $\beta \mathrm{HB}$ and leptin. (A) Weight curves for rats on the SD (filled circles, $n=5$ ), the $\mathrm{KD}$ (open circles, $n=5$ ), or the $\mathrm{CD}$ (stars, $n=4$ ) from PD 22 to PD 37. Symbols show mean body weight. Error bars show SEM if larger than the symbol. Weight curves on the SD, KD, and $\mathrm{CD}$ are significantly different from one another $(p<0.05)$. ( $B)$ Mean fat mass presented as a percentage of the total body mass excluding the head in PD 37 rats fed the $\mathrm{SD}$ (filled column, $n=5$ ), $\mathrm{KD}$ (open column, $n=5$ ), or $\mathrm{CD}$ (vertically striped bar, $n=4)$ for $16 \mathrm{~d}$. All bars differ from each other $(p<$ 0.05). Mean serum $\beta \mathrm{HB}(C)$ and leptin $(D)$ levels in PD 35-37 rats fed the SD (filled columns, $n=5$ ), the $\mathrm{KD}$ (open columns, $n=5$ ), or the $\mathrm{CD}$ (vertically striped columns, $n=4$ ) for $14-16$ d. Error bars in $B$ and $D$ show SEM. $* p$ $<0.05$ vs SD and CD.

KD do not completely explain the slower weight gain on the $\mathrm{KD}$ in rats and control mice (see below). The $o b / o b$ and $d b / d b$ mice maintained their weight gain despite having lower normalized caloric intakes on the KD (Figs. $4 B$ and $5 B$ ). ob/ob mice on the KD were ketotic since they had $360 \%$ higher midday serum $\beta \mathrm{HB}$ levels than $o b / o b$ mice on the $\mathrm{SD}$ (Fig. 4C). As expected, ob/ob mice on the KD and the SD had comparable low levels of serum leptin immunoreactivity (Fig. 4D), which probably reflects cross-immunoreactivity with the secretory form of the leptin receptor (21). Likewise, $d b / d b$ mice on the KD had $430 \%$ higher serum $\beta \mathrm{HB}$ levels and tended to have higher serum leptin levels than $d b / d b$ mice on the SD (Fig. $5 C$ and $D$ ). The much higher leptin levels in $d b / d b$ mice compared with control mice (see below) are expected (20). These results suggest that the KD requires leptin and its receptor to slow weight gain.

The background strain of the $o b / o b$ and $d b / d b$ mice did not influence the effects of the KD. C57BL/6J (background strain for $o b / o b$ mice) and C57BLKS/J (background strain for $d b / d b$ mice) mice fed the KD gained weight more slowly than those fed the SD (Figs. $4 A$ and $5 A$ ). The KD decreased normalized caloric intakes in the C57BLKS/J but not in the C57BL/6J mice (Figs. $4 B$ and $5 B$ ). The KD increased midday serum $\beta \mathrm{HB}$ levels in C57BL/6J mice by 10 -fold and in C57BLKS/J mice by sixfold (Figs. $4 C$ and $5 C$ ). The KD increased serum leptin levels by at least $130 \%$ in C57BL/6J and C57BLKS/J mice (Figs. $4 D$ and $5 D$ ). 



Figure 4. KD does not slow weight gain in leptin deficient $o b / o b$ mice. $(A)$ Weight curves for $o b / o b$ mice fed the SD (filled circles, $n=7$ ) or the KD (open circles, $n=8)$. Weight curves for control (C57BL/J6) mice fed the SD (shaded squares, $n=8-14$ ) or the KD (open squares, $n=7-14$ ). Symbols show mean body weight. Error bars show SEM if larger than symbol. Curves for the $o b / o b$ mice are comparable $(p=0.29)$ while the control mice show slower weight gain on the $\mathrm{KD}(p<0.05)$. $(B)$ Curves for caloric intake in $o b / o b$ mice on the SD and KD differ $(p<0.05)$, but the corresponding curves for control mice do not $(p=0.08)$. Symbols show mean caloric intake normalized to body weight for animals and diets in $A$. Error bars omitted for clarity. Mean serum $\beta \mathrm{HB}(C)$ and leptin $(D)$ levels at PD 44-53 in control $(w t)$ and $o b / o b$ mice fed the SD (filled columns, $n=5-14$ ) or the KD (open columns, $n=4-13$ ) for $2-3$ wk. Error bars show SEM. * $p<0.05 v s$ SD.

\section{DISCUSSION}

We reproduced the KD induced slower weight gain in epileptic children in several strains of juvenile rats and mice. Animals on the KD had higher serum leptin levels, lower insulin levels, slightly increased cortisol levels, and similar ghrelin levels compared with animals fed the SD. Only the change in leptin is consistent with slower weight gain. The failure of a KD to slow weight gain in $o b / o b$ and $d b / d b$ mice supports a role for leptin in this process.

The metabolic and hormonal effects of the KD appear to provide the brain with conflicting information regarding energy status. The high leptin levels indicate an energy-replete state and probably reflect a relative increase in fat mass, which correlates with leptin levels (20). Although another study concurs with our observation of higher leptin and lower insulin levels in KD-fed rats compared with SD-fed rats (22), we cannot explain the contrasting observations in humans with rheumatoid arthritis or type 2 diabetes who exhibit lower leptin levels on a $\operatorname{KD}(16,23)$. The lower insulin levels on a $\mathrm{KD}$ signal lower energy supplies and may result from decreased carbohydrate intake or increased insulin sensitivity $(16,22)$. The resulting change in the leptin:insulin ratio may have a role in the slower weight gain because leptin and insulin levels typically change in the same direction (8). The inverse relationship between $\beta \mathrm{HB}$ and weight suggests an important role for ketosis. The observation that rodents on nonketotic, high-fat diets gain weight faster than those on
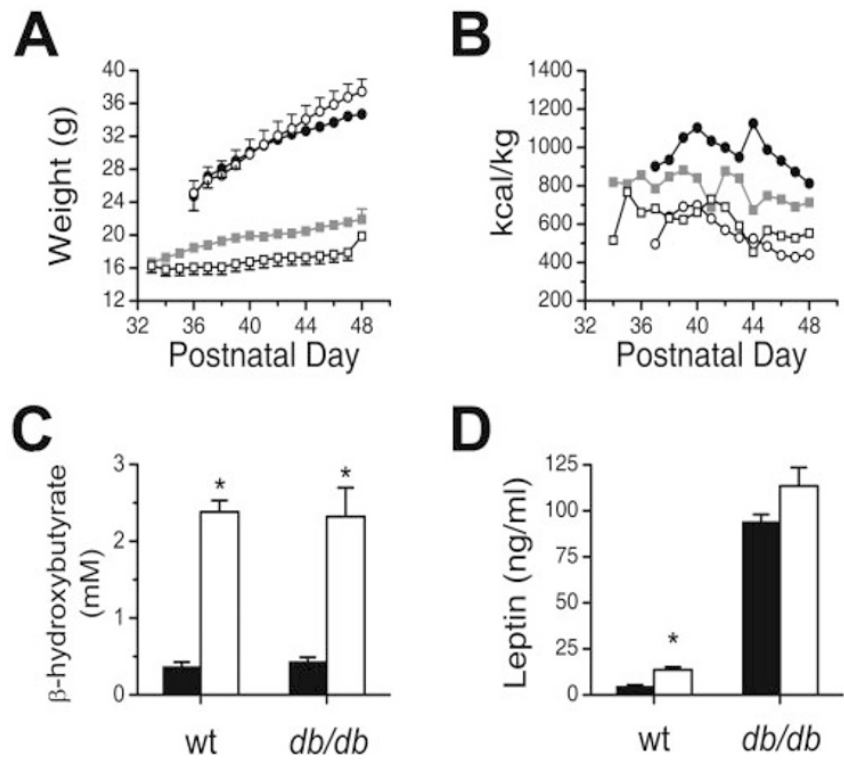

Figure 5. KD does not slow weight gain in leptin receptor deficient $d b / d b$ mice. (A) Weight curves for $d b / d b$ mice fed the SD (filled circles, $n=7$ ) or the KD (open circles, $n=8$ ). Weight curves for control (C57BLKS/J) mice fed the SD (shaded squares, $n=4-7$ ) or the KD (open squares, $n=5-8$ ). Symbols show mean body weight. Error bars show SEM if larger than symbol. Curves for the $d b / d b$ mice are comparable $(p=0.68)$ while the control mice show slower weight gain on the $\mathrm{KD}(p<0.05)$. (B) Curves for caloric intake in $d b / d b$ mice on the SD and $\mathrm{KD}$ differ $(p<0.05)$ as do the corresponding curves for control mice $(p<0.05)$. Symbols show mean caloric intake normalized to body weight for animals and diets in $A$. Error bars omitted for clarity. Mean serum $\beta \mathrm{HB}(C)$ and leptin $(D)$ levels at PD 43-51 in control ( $w t$ ) and $d b / d b$ mice fed the SD (filled columns, $n=6-7$ ) or the KD (open columns, $n=4-11$ ) for about $14 \mathrm{~d}$. Error bars show SEM. ${ }^{*} p<0.05$ vs SD.

ketotic, high-fat diets supports this idea $(22,24,25)$. Furthermore, exogenously administered $\beta \mathrm{HB}$ causes weight loss (26). Despite these observations, our results suggest that ketosis alone cannot slow weight gain because the KD caused ketosis in $o b / o b$ and $d b / d b$ mice without slowing weight gain. We do not know the reason for this discrepancy, but $\beta \mathrm{HB}$ may require leptin to alter energy balance. Overall, our results indicate that an intact leptin signaling system is necessary and that ketosis is insufficient for the KD to slow weight gain.

Our results differ from those of a recent study that found increased ghrelin levels in rats fed a KD (22). We might predict such an increase because ghrelin levels rise in association with the slower weight gain observed in rats on proteinrestricted diets (19). Although the low protein content of the KD we used may have contributed to our findings (7), the lack of a change in ghrelin and three other observations suggest that it is not the only factor involved. First, the KD maintained normal weight gain in $o b / o b$ and $d b / d b$ mice. Second, rats fed the KD showed relatively well-preserved lean mass when expressed as a percentage of body mass. Third, peak leptin levels on the CD did not exceed trough leptin levels on the SD despite the slower weight gain on the CD.

Ultimately, the KD must increase energy expenditure over energy intake to slow weight gain. The KD had variable effects on normalized caloric intake despite consistently slowing weight gain. It decreased caloric intake in C57BLKS/J, 
$d b / d b$, and $o b / o b$ mice, had no effect on caloric intake in C57BL/J6 mice, and increased caloric intake in SpragueDawley rats. Although the oily consistency of the KD chow made caloric intake measurements difficult, overall our findings suggest that the KD can decrease food intake and increase energy expenditure, which are known effects of leptin (8).

Our results support the hypothesis that the KD exerts its anticonvulsant effects by activating a variety of potassium channels via the metabolic changes it induces (27). We speculate that the KD-induced rise in serum leptin increases brain leptin levels because brain levels are proportional to serum levels (28). We expect a diffuse increase in brain levels because leptin transporters exist throughout the brain (28) and leptin increases throughout the brain when administered exogenously (29). Leptin can act as an anticonvulsant throughout the brain because leptin receptors exist throughout the brain (28) and activate calcium-activated potassium channels (9). However, the decline in insulin may counteract some of the effects of the rise in leptin because insulin activates the same channel (10). In summary, we hypothesize that the KD produces a net anticonvulsant effect in part because increased brain leptin activates potassium channels. This mechanism may be unique among antiepileptic drugs, which do not increase leptin independent of weight gain (30-32). Our results do not exclude leptin modulation of neuronal or glial energy homeostasis or other mechanisms involving the complex metabolic and cellular effects of the KD from contributing to its anticonvulsant properties (27).

Despite our focus on leptin, other neurohormones may contribute to the anticonvulsant and weight effects of the KD. For example, the increased cortisol levels we found in rats on the KD mirrors the increase seen in children on the KD and may contribute to the anticonvulsant effect of the diet (33). Others have considered the possibility that changes in neuropeptide $\mathrm{Y}$ and galanin expression contribute to the effects of the KD, but the diet does not change their brain mRNA levels (34). Rather than excluding a contribution of other neurohormones, our results indicate that studying KD-induced changes in neurohormones involved in energy homeostasis may help elucidate the mechanisms underlying the anticonvulsant and weight effects of the KD.

Acknowledgment. We thank Samuel Klein for critically reviewing the manuscript.

\section{REFERENCES}

1. Murphy P 2005 Use of the ketogenic diet as a treatment for epilepsy refractory to drug treatment. Expert Rev Neurother 5:769-775

2. Vining EP, Pyzik P, McGrogan J, Hladky H, Anand A, Kriegler S, Freeman JM 2002 Growth of children on the ketogenic diet. Dev Med Child Neurol 44:796-802

3. Liu YM, Williams S, Basualdo-Hammond C, Stephens D, Curtis R 2003 A prospective study: growth and nutritional status of children treated with the ketogenic diet. J Am Diet Assoc 103:707-712

4. Meckling KA, Gauthier M, Grubb R, Sanford J 2002 Effects of a hypocaloric, low-carbohydrate diet on weight loss, blood lipids, blood pressure, glucose tolerance, and body composition in free- living overweight women. Can J Physiol Pharmacol 80:1095-1105
5. Samaha FF, Iqbal N, Seshadri P, Chicano KL, Daily DA, McGrory J, Williams T, Williams M, Gracely EJ, Stern L 2003 A low-carbohydrate as compared with a low-fat diet in severe obesity. N Engl J Med 348:2074-2081

6. Zhao Q, Stafstrom CE, Fu DD, Hu Y, Holmes GL 2004 Detrimental effects of the ketogenic diet on cognitive function in rats. Pediatr Res 55:498-506

7. Nylen K, Likhodii S, Abdelmalik PA, Clarke J, Burnham WM 2005 A comparison of the ability of a 4:1 ketogenic diet and a 6.3:1 ketogenic diet to elevate seizure thresholds in adult and young rats. Epilepsia 46:1198-1204

8. Havel PJ 2001 Peripheral signals conveying metabolic information to the brain: short- term and long-term regulation of food intake and energy homeostasis. Exp Biol Med (Maywood) 226:963-977

9. Shanley LJ, O’Malley D, Irving AJ, Ashford ML, Harvey J 2002 Leptin inhibits epileptiform-like activity in rat hippocampal neurones via PI 3-kinase-driven activation of BK channels. J Physiol 545:933-944

10. O'Malley D, Harvey J 2004 Insulin activates native and recombinant large conductance $\mathrm{Ca}(2+)$-activated potassium channels via a mitogen-activated protein kinasedependent process. Mol Pharmacol 65:1352-1363

11. Ahima RS, Prabakaran D, Flier JS 1998 Postnatal leptin surge and regulation of circadian rhythm of leptin by feeding. Implications for energy homeostasis and neuroendocrine function. J Clin Invest 101:1020-1027

12. Ahren B 2000 Diurnal variation in circulating leptin is dependent on gender, food intake and circulating insulin in mice. Acta Physiol Scand 169:325-331

13. Cha MC, Chou CJ, Boozer CN 2000 High-fat diet feeding reduces the diurnal variation of plasma leptin concentration in rats. Metabolism 49:503-507

14. Hawkins RA, Williamson DH, Krebs HA 1971 Ketone-body utilization by adult and suckling rat brain in vivo. Biochem J 122:13-18

15. Bough KJ, Schwartzkroin PA, Rho JM 2003 Calorie restriction and ketogenic diet diminish neuronal excitability in rat dentate gyrus in vivo. Epilepsia 44:752-760

16. Boden G, Sargrad K, Homko C, Mozzoli M, Stein TP 2005 Effect of a lowcarbohydrate diet on appetite, blood glucose levels, and insulin resistance in obese patients with type 2 diabetes. Ann Intern Med 142:403-411

17. Heard CR, Frangi SM, Wright PM, McCartney PR 1977 Biochemical characteristics of different forms of protein-energy malnutrition: an experimental model using young rats. Br J Nutr 37:1-21

18. Du F, Higginbotham DA, White BD 2000 Food intake, energy balance and serum leptin concentrations in rats fed low-protein diets. J Nutr 130:514-521

19. Lee HM, Wang G, Englander EW, Kojima M, Greeley GH Jr 2002 Ghrelin, a new gastrointestinal endocrine peptide that stimulates insulin secretion: enteric distribution, ontogeny, influence of endocrine, and dietary manipulations. Endocrinology 143:185-190.

20. Maffei M, Halaas J, Ravussin E, Pratley RE, Lee GH, Zhang Y, Fei H, Kim S, Lallone R, Ranganathan S 1995 Leptin levels in human and rodent: measurement of plasma leptin and ob RNA in obese and weight-reduced subjects. Nat Med 1:11551161

21. Murakami T, Otani S, Honjoh T, Doi T, Shima K 2001 Influence of the presence of OB-Re on leptin radioimmunoassay. J Endocrinol 168:79-86

22. Kinzig KP, Scott KA, Hyun J, Bi S, Moran TH 2005 Altered hypothalamic signaling and responses to food deprivation in rats fed a low-carbohydrate diet. Obes Res 13:1672-1682

23. Fraser DA, Thoen J, Bondhus S, Haugen M, Reseland JE, Djoseland O, Forre O, Kjeldsen-Kragh J 2000 Reduction in serum leptin and IGF-1 but preserved Tlymphocyte numbers and activation after a ketogenic diet in rheumatoid arthritis patients. Clin Exp Rheumatol 18:209-214

24. Masuzaki H, Ogawa Y, Hosoda K, Kawada T, Fushiki T, Nakao K 1995 Augmented expression of the obese gene in the adipose tissue from rats fed high-fat diet. Biochem Biophys Res Commun 216:355-358

25. Ahren B 1999 Plasma leptin and insulin in C57BI/6J mice on a high-fat diet: relation to subsequent changes in body weight. Acta Physiol Scand 165:233-240

26. Davis JD, Wirtshafter D, Asin KE, Brief D 1981 Sustained intracerebroventricular infusion of brain fuels reduces body weight and food intake in rats. Science 212:81-83

27. Vamecq J, Vallee L, Lesage F, Gressens P, Stables JP 2005 Antiepileptic popular ketogenic diet: emerging twists in an ancient story. Prog Neurobiol 75:1-28

28. Banks WA 2004 The many lives of leptin. Peptides 25:331-338

29. Fliedner S, Schulz C, Lehnert H 2006 Brain uptake of intranasally applied radioiodinated leptin in Wistar rats. Endocrinology 147:2088-2094

30. York DA, Singer L, Thomas S, Bray GA 2000 Effect of topiramate on body weight and body composition of Osborne-Mendel rats fed a high-fat diet: alterations in hormones, neuropeptide, and uncoupling-protein mRNAs. Nutrition 16:967-975

31. Greco R, Latini G, Chiarelli F, Iannetti P, Verrotti A 2005 Leptin, ghrelin, and adiponectin in epileptic patients treated with valproic acid. Neurology 65:1808-1809

32. Himmerich H, Koethe D, Schuld A, Yassouridis A, Pollmacher T 2005 Plasma levels of leptin and endogenous immune modulators during treatment with carbamazepine or lithium. Psychopharmacology (Berl) 179:447-451

33. Fraser DD, Whiting S, Andrew RD, Macdonald EA, Musa-Veloso K, Cunnane SC 2003 Elevated polyunsaturated fatty acids in blood serum obtained from children on the ketogenic diet. Neurology 60:1026-1029

34. Tabb K, Szot P, White SS, Liles LC, Weinshenker D 2004 The ketogenic diet does not alter brain expression of orexigenic neuropeptides. Epilepsy Res 62:35-39 